\title{
Quantization of the planar affinely-rigid body
}

\author{
Agnieszka MARTENS \\ Institute of Fundamental Technological Research, \\ Polish Academy of Sciences \\ Świętokrzyska 21, 00-049 Warsaw, Poland \\ E-mail: amartens@ippt.gov.pl
}

This article is part of the Proceedings titled "Geometrical Mathods in Physics: Bialowieza XXI and XXII"

\begin{abstract}
This paper is a continuation of [1] where the classical model was analyzed. Discussed are some quantization problems of two-dimensional affinely rigid body with the double dynamical isotropy. Considered are highly symmetric models for which the variables can be separated. Some explicit solutions are found using the Sommerfeld polynomial method.
\end{abstract}

\section{Introduction}

In [1] we discussed the classical dynamics of the planar affinely-rigid body. The actionangle analysis and discussion of degeneracy as well as the quasiclassical Bohr-Sommerfeld quantization were also presented.

The two-dimensional problem may be effectively studied and may have some practical applications both in macroscopic elasticity and in microscopic physical problems, e.g. on the molecular level. There exist then potential models which are simultaneously physically realistic and analytically treatable. On the classical level they are completely integrable and may show some degeneracy properties following from hidden symmetries. We investigate here the Schrödinger quantization procedure for such an object. We follow the standard procedure of quantization in Riemannian manifolds [2], i.e. we use the $L^{2}$-Hilbert space of wave functions in the sense of the usual Riemannian measure (volume element). The classical kinetic energy is replaced by the corresponding quantum expression based on the Laplace-Beltrami operator. The separation of variables is performed and then the corresponding one-dimensional Schrödinger equations are solved using the Sommerfeld polynomial method [3].

\section{Schrödinger quantization}

Let us present now general ideas of the rigorous quantum-mechanical version of the model investigated in [1]. We consider the two-dimensional body subject to constraints which confine its deformative behavior to homogeneous strains. We do not take into account 
the motion of the center of mass. When translational degrees of freedom are neglected, the configuration space becomes identical with the linear group: $Q=G L(2, \mathbb{R})$-the group of non-singular real $2 \times 2$ matrices. We will use the Cartesian and polar coordinates (mentioned in $[4,5,1])$ to solve the stationary Schrödinger equation.

We use the Hilbert space $L^{2}(Q, \tilde{\mu})$ with the usual scalar product:

$$
\left\langle\Psi_{1} \mid \Psi_{2}\right\rangle=\int \bar{\Psi}_{1} \Psi_{2} d \tilde{\mu}
$$

where $\Psi_{1}, \Psi_{2}$ are wave functions and $\tilde{\mu}$ is the usual Riemannian measure. Therefore, in the Cartesian and polar coordinates we have respectively:

$$
\begin{aligned}
& d \tilde{\mu}(\theta, \psi, \alpha, \beta)=\sqrt{\left|\operatorname{det}\left[G_{i j}\right]\right|} d \theta d \psi d \alpha d \beta, \quad\left[G_{i j}\right]=\left[\begin{array}{cccc}
\alpha^{2}+\beta^{2} & \beta^{2}-\alpha^{2} & 0 & 0 \\
\beta^{2}-\alpha^{2} & \alpha^{2}+\beta^{2} & 0 & 0 \\
0 & 0 & 1 & 0 \\
0 & 0 & 0 & 1
\end{array}\right], \\
& d \tilde{\mu}(\theta, \psi, r, \varphi)=\sqrt{\left|\operatorname{det}\left[G_{i j}\right]\right|} d \theta d \psi d r d \varphi, \quad\left[G_{i j}\right]=\left[\begin{array}{cccc}
r & -r \cos \varphi & 0 & 0 \\
-r \cos \varphi & r & 0 & 0 \\
0 & 0 & \frac{1}{4 r} & 0 \\
0 & 0 & 0 & \frac{r}{4}
\end{array}\right] .
\end{aligned}
$$

The Hamiltonian operator $\hat{H}$ is given by the expression:

1. Cartesian coordinates:

$$
\hat{H}=\hat{H}_{\alpha}+\hat{H}_{\beta}=-\frac{\hbar^{2}}{2 \mu} \Delta+V(\alpha, \beta),
$$

where

$$
\begin{aligned}
& \hat{H}_{\alpha}=\frac{1}{2 \mu}\left(\frac{1}{\alpha^{2}}(\hat{S}-\hat{\Sigma})^{2}-\hbar^{2}\left(\frac{\partial^{2}}{\partial \alpha^{2}}+\frac{1}{\alpha} \frac{\partial}{\partial \alpha}\right)\right)+V_{\alpha}(\alpha), \\
& \hat{H}_{\beta}=\frac{1}{2 \mu}\left(\frac{1}{\beta^{2}}(\hat{S}+\hat{\Sigma})^{2}-\hbar^{2}\left(\frac{\partial^{2}}{\partial \beta^{2}}+\frac{1}{\beta} \frac{\partial}{\partial \beta}\right)\right)+V_{\beta}(\beta),
\end{aligned}
$$

and $\hat{S}=\frac{\hbar}{i} \frac{\partial}{\partial \theta}$ is the spin operator, the generator of spatial rotations, and $\hat{\Sigma}=\frac{\hbar}{i} \frac{\partial}{\partial \psi}$ is the "vorticity" operator, the generator of material rotations. Operators $\hat{H}_{\alpha}, \hat{H}_{\beta}, \hat{S}$, $\hat{\Sigma}$ are the quantum constants of motion. They also commute with each other (they represent co-measurable physical quantities).

2. Polar coordinates:

$$
\hat{H}=\hat{H}_{r}+\frac{\hat{h}_{\varphi}}{r}=-\frac{\hbar^{2}}{2 \mu} \Delta+V(r, \varphi),
$$

where

$$
\begin{aligned}
& \hat{H}_{r}=-\frac{2 \hbar^{2}}{\mu}\left(r \frac{\partial^{2}}{\partial r^{2}}+2 \frac{\partial}{\partial r}\right)+V_{r}(r) \\
& \hat{h}_{\varphi}=\frac{1}{2 \mu}\left(\frac{1}{\sin ^{2} \varphi}\left(\hat{S}^{2}+\hat{\Sigma}^{2}+2 \hat{S} \hat{\Sigma} \cos \varphi\right)-4 \hbar^{2}\left(\frac{\partial^{2}}{\partial \varphi^{2}}+\cot \varphi \frac{\partial}{\partial \varphi}\right)\right)+V_{\varphi}(\varphi) .
\end{aligned}
$$

In these coordinates, the operators $\hat{H}, \hat{h}_{\varphi}, \hat{S}, \hat{\Sigma}$ are the quantum constants of motion. 
We restrict ourselves to the Stäckel-type potentials [1] and $\Delta$ is the Laplace-Beltrami operator corresponding to the metric $G$ and its action on the wave function is given by the following expression:

$$
\Delta \Psi=G^{i j} \nabla_{i} \nabla_{j} \Psi=\frac{1}{\sqrt{|G|}} \sum_{i, j} \frac{\partial}{\partial x^{i}}\left(\sqrt{|G|} G^{i j} \frac{\partial \Psi}{\partial x_{j}}\right),
$$

where $\nabla$ denotes the operator of covariant differentiation. Thus, after some calculations we obtain:

1. Cartesian coordinates:

$$
\begin{aligned}
& \Delta \Psi=\frac{\partial^{2} \Psi}{\partial \alpha^{2}}+\frac{\partial^{2} \Psi}{\partial \beta^{2}}+\frac{1}{\alpha} \frac{\partial \Psi}{\partial \alpha}+\frac{1}{\beta} \frac{\partial \Psi}{\partial \beta}+\left(\frac{1}{4 \alpha^{2}}+\frac{1}{4 \beta^{2}}\right)\left(\frac{\partial^{2} \Psi}{\partial \theta^{2}}+\frac{\partial^{2} \Psi}{\partial \psi^{2}}\right)+ \\
& +\left(\frac{1}{2 \beta^{2}}-\frac{1}{2 \alpha^{2}}\right) \frac{\partial^{2} \Psi}{\partial \theta \partial \psi}
\end{aligned}
$$

A complete system of solutions of the stationary Schrödinger equation $\hat{H} \Psi=E \Psi$ has the form:

$$
\Psi(\theta, \psi, \alpha, \beta)=\Theta(\theta) \Upsilon(\psi) A(\alpha) B(\beta),
$$

where $\Theta(\theta)=e^{i m \theta}, \Upsilon(\psi)=e^{i l \psi}(m, l$ are integers) and $A(\alpha), B(\beta)$ are the purely deformative wave functions.

2. Polar coordinates:

$$
\begin{gathered}
\Delta \Psi=\frac{1}{r \sin ^{2} \varphi}\left(\frac{\partial^{2} \Psi}{\partial \theta^{2}}+\frac{\partial^{2} \Psi}{\partial \psi^{2}}\right)+\frac{2 \cos \varphi}{r \sin ^{2} \varphi} \frac{\partial^{2} \Psi}{\partial \theta \partial \psi}+4 r \frac{\partial^{2} \Psi}{\partial r^{2}}+8 \frac{\partial \Psi}{\partial r}+\frac{4}{r}\left(\frac{\partial^{2} \Psi}{\partial \varphi^{2}}+\cot \varphi \frac{\partial \Psi}{\partial \varphi}\right) \\
\Psi(\theta, \psi, r, \varphi)=\Theta(\theta) \Upsilon(\psi) R(r) F(\varphi)
\end{gathered}
$$

where $R(r), F(\varphi)$ are the deformative wave functions.

Hence, the stationary Schrödinger equation with an arbitrary potentials $V(\alpha, \beta)=V_{\alpha}(\alpha)+$ $V_{\beta}(\beta), V(r, \varphi)=V_{r}(r)+\frac{V_{\varphi}(\varphi)}{r}$ leads after the standard separation procedure to the following system of one-dimensional eigenequations:

1. Cartesian coordinates:

$$
\begin{aligned}
& \frac{\partial^{2} A(\alpha)}{\partial \alpha^{2}}+\frac{1}{\alpha} \frac{\partial A(\alpha)}{\partial \alpha}-\frac{(m-l)^{2}}{4 \alpha^{2}} A(\alpha)+\frac{2 \mu}{\hbar^{2}}\left(E_{\alpha}-V_{\alpha}(\alpha)\right) A(\alpha)=0, \\
& \frac{\partial^{2} B(\beta)}{\partial \beta^{2}}+\frac{1}{\beta} \frac{\partial B(\beta)}{\partial \beta}-\frac{(m+l)^{2}}{4 \beta^{2}} B(\beta)+\frac{2 \mu}{\hbar^{2}}\left(E_{\beta}-V_{\beta}(\beta)\right) B(\beta)=0,
\end{aligned}
$$

where $E_{\alpha}$ and $E_{\beta}$ are fixed values of energy. 
2. Polar coordinates:

$$
\begin{aligned}
& \frac{\partial^{2} R(r)}{\partial r^{2}}+\frac{2}{r} \frac{\partial R(r)}{\partial r}+\frac{\mu}{2 \hbar^{2} r}\left(E-V_{r}(r)-\frac{e_{\varphi}}{r}\right) R(r)=0 \\
& \frac{\partial^{2} F(\varphi)}{\partial \varphi^{2}}+\cot \varphi \frac{\partial F(\varphi)}{\partial \varphi}-\left(\frac{m^{2}+l^{2}+2 m l \cos \varphi}{4 \sin ^{2} \varphi}+\frac{\mu}{2 \hbar^{2}}\left(V_{\varphi}(\varphi)-e_{\varphi}\right)\right)=0
\end{aligned}
$$

where $e_{\varphi}$ is a separation constant and $E$ is a fixed value of the energy.

It is natural to expect that for potentials discussed in [1] the resulting Schrödinger equations should be rigorously solvable in terms of some standard special functions. The most convenient way of solving them is to use the Sommerfeld polynomial method [3].

\section{Sommerfeld polynomial method}

In this method the solutions are expressed by the usual or confluent Riemann $P$-functions. They are deeply related to the hypergeometric functions (respectively usual $F_{1}$ or confluent $F_{2}$ ). If the usual convergence demands are imposed, then the hypergeometric functions become polynomials and our solutions are expressed by elementary functions. At the same time the energy levels and separation constants are expressed by the eigenvalues of the corresponding operators. There exists some special class of potentials to which the Sommerfeld polynomial method is applicable. The restriction to solutions expressible in terms of Riemann $P$-functions is reasonable, because this class of functions is well investigated and many special functions used in physics may be expressed by them. There is also an intimate relationship between these functions and representations of Lie groups.

\section{Some rigorously solvable models}

There exist potentials given by the formula $[4,5]: V=\frac{A}{\alpha^{2}}+\frac{B}{\beta^{2}}+C\left(\alpha^{2}+\beta^{2}\right)$, where $A$, $B, C$ are constants, which could lead to Schrödinger equation separable simultaneously in Cartesian and polar coordinate systems. This simultaneous separability usually leads to some degeneracy of the problem.

The equations (2.9) and (2.10) may be solved only when the explicit forms of potentials $V(\alpha, \beta)$ and $V(r, \varphi)$ are specified. It is clear that simple solutions in terms of known special functions may be expected only when the potential has some particular geometric interpretation. For example, this is the case when the corresponding classical problem is degenerate and has some hidden symmetries.

\subsection{Cartesian model}

Here we consider the model of the Cartesian potential, i.e.

$$
V(\alpha, \beta)=\frac{C}{4}\left(\alpha^{2}+\frac{4}{\alpha^{2}}\right)+\frac{C}{4} \beta^{2}, \quad C>0 .
$$

Applying the Sommerfeld polynomial method we obtain the energy levels $E=E_{\alpha}+E_{\beta}$ as follows:

$$
E=\frac{1}{2 \sqrt{2}} \hbar \omega\left(4 n+2+|m+l|+\sqrt{(m-l)^{2}+\frac{8 C \mu}{\hbar^{2}}}\right),
$$


where

$$
E_{\alpha}=\frac{\hbar \omega}{2 \sqrt{2}}\left(4 n_{\alpha}+1+\sqrt{(m-l)^{2}+\frac{8 C \mu}{\hbar^{2}}}\right), \quad E_{\beta}=\frac{\hbar \omega}{2 \sqrt{2}}\left(4 n_{\beta}+1+|m+l|\right),
$$

and $\omega=\sqrt{\frac{C}{\mu}}, n=0,1, \ldots ; m, l=0, \pm 1, \ldots$ The energy in (4.2) depends on an integer combination of the quantum numbers, i.e. $n=n_{\alpha}+n_{\beta}$. After some calculations we obtain the wave functions $A(\alpha)$ and $B(\beta)$ in the form:

$$
\begin{aligned}
& A(\alpha)=\alpha^{\chi} \kappa^{\frac{1}{4}+\frac{\chi}{2}} e^{-\frac{\kappa}{2} \alpha^{2}} F_{2}\left(-n_{\alpha} ; 1+\chi ; \kappa \alpha^{2}\right), \\
& B(\beta)=\beta^{\gamma} \kappa^{\frac{1}{4}+\frac{\gamma}{2}} e^{-\frac{\kappa}{2} \beta^{2}} F_{2}\left(-n_{\beta} ; 1+\gamma ; \kappa \beta^{2}\right),
\end{aligned}
$$

where $\chi=\sqrt{(m-l)^{2}+\frac{8 C \mu}{\hbar^{2}}}, \kappa=\sqrt{\frac{C \mu}{2 \hbar^{2}}}, \gamma=\frac{1}{4}|m+l|$.

\subsection{Polar model}

Next we consider the model of the polar potential, i.e.

$$
V(r, \varphi)=\frac{C}{4}\left(r+\frac{4}{r}\right)+\frac{C}{r} \operatorname{tg}^{2} \frac{\varphi}{2}, \quad C>0 .
$$

Here we obtain the following expressions for the spectrum of eigenvalues of the constant $e_{\varphi}$ and the energy levels $E$ as follows:

$$
E=\frac{1}{2 \sqrt{2}} \hbar \omega\left(4 n_{r}+\sqrt{4+16 e_{\varphi}+\frac{8 C \mu}{\hbar^{2}}}\right)
$$

where $e_{\varphi}=\frac{1}{16}\left(\left(4 n_{\varphi}+2+|m+l|+\sqrt{(m-l)^{2}+\frac{8 C \mu}{\hbar^{2}}}\right)^{2}-4-\frac{8 C \mu}{\hbar^{2}}\right)$. Finally we obtain the energy spectrum in the following form:

$$
E=\frac{1}{2 \sqrt{2}} \hbar \omega\left(4 n+2+|m+l|+\sqrt{(m-l)^{2}+\frac{8 C \mu}{\hbar^{2}}}\right) .
$$

The energy in (4.7) depends on an integer combination of the quantum numbers, i.e. $n=n_{r}+n_{\varphi}$. The functions $R(r)$ and $F(\varphi)$ are as follows:

$$
\begin{aligned}
& R(r)=r^{-\frac{1}{2}(1-\xi)} \epsilon^{\frac{1}{2}} e^{-\frac{\epsilon}{2} r} F_{2}\left(-n_{r} ; 1+\xi ; r \epsilon\right), \\
& F(\varphi)=\left(\cos \frac{\varphi}{2}\right)^{\frac{\chi}{2}}\left(\sin \frac{\varphi}{2}\right)^{2 \gamma} F_{1}\left(-n_{\varphi}, n_{\varphi}+1+2 \gamma+\frac{1}{2} \chi ; 1+\frac{1}{2} \chi ; \cos ^{2} \frac{\varphi}{2}\right),
\end{aligned}
$$

where $\xi=\sqrt{1+4 e_{\varphi}+\frac{2 \mu^{2} \omega^{2}}{\hbar^{2}}}$ and $\epsilon=\frac{\sqrt{\mu \kappa}}{\hbar^{2}}$. 


\section{Conclusions}

Separability of the stationary Schrödinger equation in two different coordinate systems has to do with degeneracy of the problem. Due to the lack of complete degeneracy in the corresponding classical problems, topological closures of classical trajectories are threedimensional tori. The systems are one-fold degenerate. The quantum numbers labeling the energy levels cannot be combined into a single quantum number, i.e. there is no quantum degeneracy with respect to them.

Acknowledgments. Special thanks are to Professor Jan J. Sławianowski for his help during my work on this article. I am also indebted to University in Białystok, Institute of Fundamental Technological Research and Polish Committee of Scientific Research (KBN) for the financial support of my participation in the XXIInd Workshop on Geometric Methods in Physics. I am very grateful to Professor A. Odzijewicz for the invitation and his hospitality. The paper was prepared within the KBN grant 8T07A04720.

\section{References}

[1] Martens A, Hamiltonian dynamics of planar affinely-rigid body, J. of Nonlin. Math. Phys., in these proceedings.

[2] Mackey G. W, The mathematical Foundation of Quantum Mechanics, W. A. Benjamin, Inc., New York, Amsterdam, 1963.

[3] Rubinowicz W, Quantum Mechanics, PWN, Warszawa 1968.

[4] Sławianowski J J, Analytical Mechanics of Deformable Bodies, PWN, Warszawa-Poznań, 1982. (in Polish).

[5] Sławianowski J J, ZAAM, 62, 229, 1982. 